\title{
Perfil epidemiológico das internações por transtornos mentais e comportamentais devido ao uso de álcool no município de Patos de Minas, Minas Gerais
}

\author{
Epidemiological profile of hospitalizations for mental and behavioral disorders due to alcohol use \\ in the municipality of Patos de Minas, Minas Gerais \\ Perfil epidemiológico de las hospitalizaciones por trastornos mentales y del comportamiento por \\ consumo de alcohol en el município de Patos de Minas, Minas Gerais
}

Laize Rodrigues da Fonseca ORCID: https://orcid.org/0000-0001-9028-6302 Centro Universitário de Patos de Minas, Brasil E-mail: fonsecarlaize@gmail.com

Barbara Dayane Ribeiro ORCID: https://orcid.org/0000-0001-7706-3390 Centro Universitário de Patos de Minas, Brasil E-mail: barbararibeiro@unipam.edu.br

Camila Adriane Almeida Silva ORCID: https://orcid.org/0000-0003-3213-4786 Centro Universitário de Patos de Minas, Brasil E-mail: camilaadriane@unipam.edu.br

Eduarda Rocha Santos

ORCID: https://orcid.org/0000-0003-0910-4781 Centro Universitário de Patos de Minas, Brasil E-mail: eduardarocha@unipam.edu.br

Fabiana de Souza Silva

ORCID: https://orcid.org/0000-0001-7541-7829

Centro Universitário de Patos de Minas, Brasil E-mail: fabianasouza@unipam.edu.br

Lara Fernanda Papalardo Brandão ORCID: https://orcid.org/0000-0003-4097-5649 Centro Universitário de Patos de Minas, Brasil E-mail: larabrandao@unipam.edu.br

Laura Santos Oliveira

ORCID: https://orcid.org/0000-0002-6555-8465 Centro Universitário de Patos de Minas, Brasil E-mail: laurasoliveira@unipam.edu.br

Sara Claudino dos Santos

ORCID: https://orcid.org/0000-0001-5290-6339

Centro Universitário de Patos de Minas, Brasil E-mail: saraclaudiano@unipam.edu.br

\begin{abstract}
Resumo
Introdução: O uso abusivo de álcool possui efeito nocivo em diversas fases da vida, gerando transtornos mentais e comportamentais de suma importância. Aliado a isso, há um número exorbitante de indivíduos que ingerem álcool com frequência e podem ou não resultar em um uso abusivo. Diante disso, dados atuais demonstram a elevada mortalidade associada ao uso nocivo de álcool o que gerou muitas dúvidas sobre qual seria o perfil dessa população. Objetivo: identificar os aspectos envolvidos na epidemiologia do uso nocivo de álcool no município de Patos de Minas. Metodologia: O presente trabalho consiste em um estudo epidemiológico descritivo, retrospectivo com abordagem de natureza quantitativa. Foi realizada uma pesquisa de dados no Departamento de Informática do Sistema Único de Saúde (DATASUS), analisando todos os casos de internações por transtornos mentais e comportamentais devido ao uso de álcool no município de Patos de Minas (MG), compreendidos entre o período de 2010 a 2020. Discussão: A parcela mais expressiva da população foram os homens, em idade economicamente ativa. Esse fato evidencia o perfil epidemiológico mais prevalente, mesmo havendo uma relativa subnotificação. Considerações finais: Tendo em vista o perfil epidemiológico mais expressivo, fazem-se necessárias ações educativas em saúde que visem modificar esse painel epidemiológico e mitigando o aumento de casos e possíveis riscos.

Palavras-chave: Epidemiologia; Álcool; Internações; Patos de Minas; Hospitalização; Distúrbios psiquiátricos; Uso de serviços de saúde.
\end{abstract}




\begin{abstract}
Introduction: The abusive use of alcohol has harmful effects at different stages of life, generating mental and behavioral disorders of paramount importance. In addition, there is an exorbitant number of individuals who drink alcohol frequently and that may or may not result in abuse. Therefore, current data demonstrate the high mortality associated with the harmful use of alcohol, which raised many questions about the profile of this population. Objective: Identify the aspects involved in the epidemiology of the harmful use of alcohol in the city of Patos de Minas. Methodology: The present work consists of a descriptive, retrospective epidemiological study with a quantitative approach. A data research was carried out in the Informatics Department of the Unified Health System (DATASUS), analyzing all cases of hospitalizations for mental and behavioral disorders due to alcohol use in the city of Patos de Minas (MG), between 2010 and 2020. Discussion: The most expressive portion of the population were working age men. This fact highlights the most prevalent epidemiological profile, even with relative underreporting. Final considerations: In view of the more expressive epidemiological profile, health educational measures that aim to modify this epidemiological panel and mitigate the increase in cases and possible risks are necessary.

Keywords: Epidemiology; Alcohol; Admissions; Ducks from Minas; Hospitalization; Psychiatric disorders; Use of health services.

\section{Resumen}

Introducción: El uso abusivo del alcohol tiene efectos nocivos en las diferentes etapas de la vida, generando trastornos mentales y del comportamiento de suma importancia. Además, hay un número exorbitante de personas que beben alcohol con frecuencia y pueden o no resultar en abuso. Por tanto, los datos actuales demuestran la alta mortalidad asociada al uso nocivo del alcohol, lo que generó muchas dudas sobre el perfil de esta población. Objetivo: identificar los aspectos involucrados en la epidemiología del uso nocivo de alcohol en la ciudad de Patos de Minas. Metodología: El presente trabajo consiste en un estudio epidemiológico descriptivo, retrospectivo con enfoque cuantitativo. Se realizó una búsqueda de datos en el Departamento de Informática del Sistema Único de Salud (DATASUS), analizando todos los casos de hospitalizaciones por trastornos mentales y del comportamiento por consumo de alcohol en la ciudad de Patos de Minas (MG), entre el período de 2010 a 2020. Discusión: La porción más expresiva de la población fueron los hombres, en edad laboral. Este hecho pone de relieve el perfil epidemiológico más prevalente, incluso con un subregistro relativo. Consideraciones finales: Ante el perfil epidemiológico más expresivo, son necesarias acciones educativas en salud que tengan como objetivo modificar este panel epidemiológico y mitigar el aumento de casos y posibles riesgos.
\end{abstract}

Palabras clave: Epidemiología; Alcohol; Admisiones; Patos de Minas; Hospitalización; Desórdenes psiquiátricos; Uso de servicios de salud.

\title{
1. Introdução
}

O abuso de álcool pode desencadear transtornos mentais e comportamentais em todas as idades, entretanto seu efeito é particularmente sensível na adolescência, devido ao comprometimento do desenvolvimento de funções ligadas à maturação biológica do sistema nervoso central (Rocha, Oliveira, Brum, Cavalcante \& Machado, 2015). Esses danos psicológicos podem continuar presentes na vida adulta, se tornando mais graves. Essas pessoas possuem vivências específicas de sofrimento físico e psicológico e, por isso, necessitam de prevenção e intervenções mais eficazes quanto à reestruturação de suas vidas sem o álcool (Rocha et al., 2015).

O Manual de Diagnósticos e Estatístico de Transtornos Mentais (DSM-5), publicado em 2014, atribui sintomas importantes a intoxicação por álcool, como comportamento sexual ou agressivo inadequado, humor instável, julgamento prejudicado e comprometimento no funcionamento social ou profissional e, caso a intoxicação seja intensa, pode resultar em coma potencialmente letal. Ademais, existe uma relação intrínseca entre a intoxicação por álcool e episódios de violência, incluindo brigas, abusos domésticos e homicídios (Castro, 2014), assim como acidentes de trânsito, sendo que a Pesquisa Nacional de Saúde (PNS) estimou que 17\% dos indivíduos conduzem veículos motorizados após ingestão de bebidas alcoólicas (Instituto Brasileiro de Geografia e Estatística [IBGE], 2019).

O Global Status Report on Alcohol and Health estima que em 2016, o uso nocivo de álcool resultou em cerca de 3 milhões de mortes (5,3\% do total mortes). A mortalidade resultante do consumo de álcool é maior do que a causada por doenças como tuberculose, vírus da imunodeficiência humana (HIV) e síndrome da imunodeficiência adquirida (AIDS) e diabetes. Cerca de 2,3 bilhões de pessoas com 15 anos ou mais bebem atualmente, e em todo o mundo, mais de um quarto 
(26,5\%) de todos os jovens de 15 a 19 anos bebem, no valor de 155 milhões de adolescentes (World Health Organization [WHO], 2018).

Além disso, o relatório indica que o consumo de 60 gramas ou mais de álcool puro de recorrência mensal mínima, atinge seu pico na idade de 20-24 anos (WHO, 2018), sendo essas situações caracterizadas como episódios etílicos excessivos (heavy drinking episodes - HED). Diferenças de gênero parecem se tornar muito menos relevantes recentemente, sobretudo durante a adolescência e no início da idade adulta (DSM-5).

A luz do exposto, percebe-se necessário conhecer as características particulares quanto a esses indivíduos, principalmente em âmbito regional, para inspirar a ampliação dos recursos já existentes e até mesmo a criação de novas estratégias mais eficientes e satisfatórias. Portanto, esse trabalho possui como objetivo identificar a epidemiologia dos indivíduos internados por transtornos mentais e comportamentais devido ao uso de álcool, considerando aspectos como idade, sexo, gastos, dias de permanência e taxa de mortalidade, no período de 2010 a 2020, na cidade de Patos de Minas, em Minas Gerais.

\section{Metodologia}

Trata-se de um estudo epidemiológico descritivo, retrospectivo com abordagem de natureza quantitativa. Os dados foram obtidos por meio de consulta ao Departamento de Informática do Sistema Único de Saúde (DATASUS), no endereço eletrônico (http://www.datasus.gov.br) nas através da consulta aos bancos de dados do Sistema de Internação Hospitalar (SIH/SUS). O levantamento de dados ocorreu no período entre os meses de Setembro a Novembro de 2021. Para evitar erros de retardo de notificação, optou-se por analisar os dados disponíveis até 2020, último ano em que constavam os dados completos.

Constituída por todos os casos de internações por transtornos mentais e comportamentais devido ao uso de álcool no município de Patos de Minas (MG), diagnosticados e registrados no período de 2010 a 2020. Foram analisados os seguintes parâmetros: faixa etária, sexo, cor/raça, estabelecimento e caráter de atendimento. Como também, dias de permanência, taxa de mortalidade e o valor total das internações.

Os dados foram inicialmente tabulados no Microsoft Office Excel e foram tratados estatisticamente (em termos de média, correlações e números absolutos), por meio do programa Statistical Package for the Social Sciences (SPSS) versão 20.0. Por se tratar de um banco de domínio público, não foi necessário submeter o projeto ao Comitê de Ética em Pesquisa.

\section{Resultados}

Fora notificado entre o período de 2010 a 2020 um total de 95 internações, segundo a lista de morbidades do do CID10 capítulo 05, sendo o maior percentual nos anos de 2010 (22\%), 2014 (14\%) e 2013 (11,5\%). Quando analisado o sexo houve um predomínio das internações pelo sexo masculino (72,63\%) quando comparado ao feminino. A partir dos resultados obtidos observou-se que as internações foram mais frequentes no sexo masculino, n 69 (72,6\%) já no sexo feminino houve 25 internações $(17,4 \%)$. Quando analisada a raça a maioria dos pacientes estão alocados em um grupo sem classificação de cor (n 43), seguido pela raça branca (n38), preta (n 5) e os grupos parda, amarela e indígena juntos somam 9 internações no período observado. 
Research, Society and Development, v. 11, n. 1, e19311124640, 2022

(CC BY 4.0) | ISSN 2525-3409 | DOI: http://dx.doi.org/10.33448/rsd-v11i1.24640

Tabela 1: Internações por ano de processamento, no período de 2010 a 2020.

\begin{tabular}{ccc}
\hline Ano processamento & Internações & Percentual \\
\hline 2010 & 21 & $22 \%$ \\
2011 & 8 & $8 \%$ \\
2012 & 9 & $9 \%$ \\
2013 & 11 & $12 \%$ \\
2014 & 13 & $14 \%$ \\
2015 & 5 & $5 \%$ \\
2016 & 8 & $8 \%$ \\
2017 & 4 & $4 \%$ \\
2018 & 2 & $2 \%$ \\
2019 & 7 & $7 \%$ \\
2020 & 7 & $7 \%$ \\
\hline Total & 95 & $100 \%$
\end{tabular}

Fonte: Ministério da Saúde - Sistema de Informações Hospitalares do SUS (SIH/SUS) (2021).

Em relação a faixa etária, observou-se que o maior número de internações ocorreu com pacientes de 40 a 49 anos (35,7\%), pacientes de 30 a 39 anos somaram 24, 2\% das internações; 50 a 59 anos representou 20\%, 20 a 29 anos corresponde a 8,4\% das internações, 60 a 69 anos 7,3\% e de 1 a 19 anos 3,1\% das internações no período analisado.

Figura 1: Internações por Faixa Etária, 2010 a 2020.

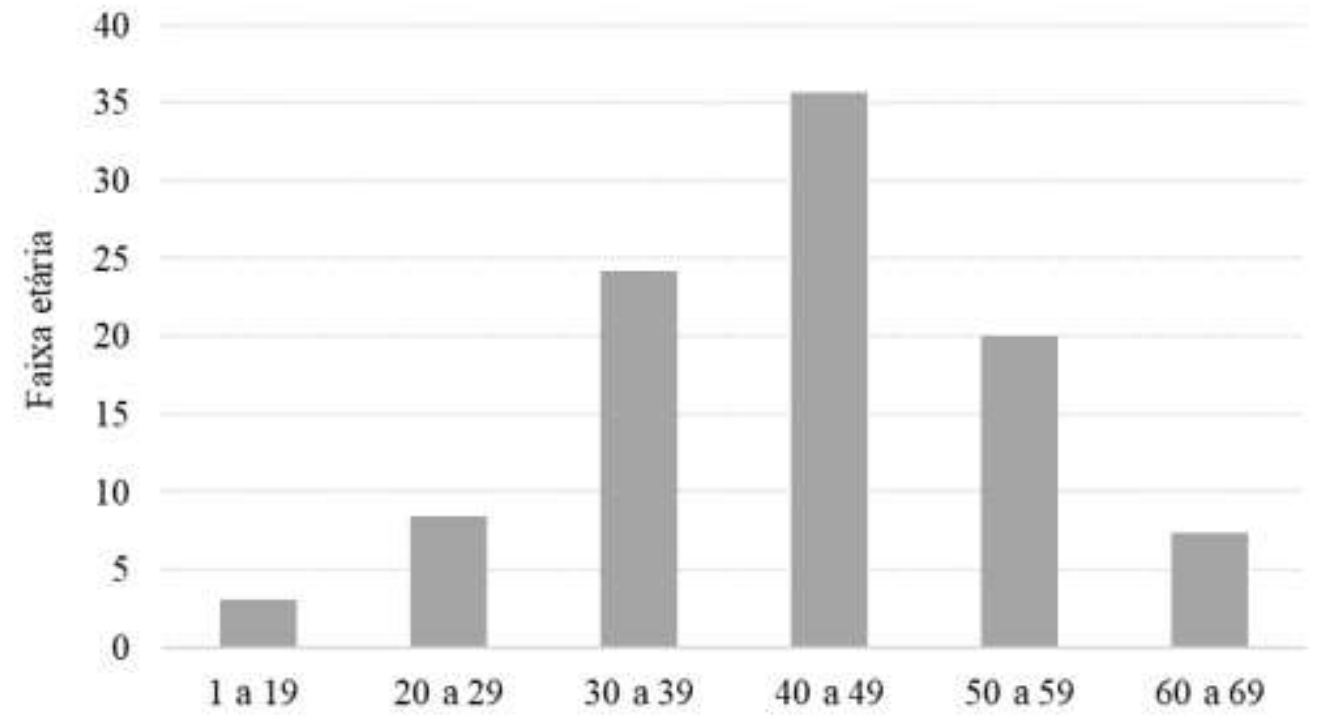

Fonte: Ministério da Saúde - Sistema de Informações Hospitalares do SUS (SIH/SUS) (2021).

Em relação ao valor total das internações observou-se uma grande variação, cujo valor registrado em 2010 foi de R\$ 9.954,21 passando para $\mathrm{R} \$ 1.370,83 \mathrm{em} 2015$ e posteriormente $\mathrm{R} \$ 6.978,18 \mathrm{em} 2020$. Os maiores custos no período foram no ano de 2014 chegando a R \$11.403,07. 
Em relação aos valores por faixa etária, o grupo com maior número de internações (40 a 49 anos), totalizou entre o período de 2010 a 2020 um gasto de 14179, 17 reais em relação aos serviços hospitalares prestados. E ainda, obtivemos que o valor médio das consultas em tal período é de 688,21 reais, sendo 752,49 reais para o sexo masculino e 517,60 reais no sexo feminino, com média de permanência hospital de 9,7 e 11,1 dias no sexo masculino e feminino, respectivamente. Sendo que se tratando da média de permanência hospitalar, o maior tempo de internação foi 15,7 dias em 2012.

Observou-se ainda, que 2 pacientes, do sexo masculino evoluíram para morte, uma no ano de 2014 e outra no ano de 2019. Resultando, assim, em uma taxa de mortalidade igual a 2,1.

Tabela 1: Internações por tempo de permanência hospitalar, óbitos e taxa de mortalidade, 2010 a 2020.

\begin{tabular}{ccccc}
\hline $\begin{array}{c}\text { Ano } \\
\text { atendimento }\end{array}$ & Dias_permanência & Média_permanência & Óbitos & Taxa_mortalidade \\
\hline 2010 & 215 & 9,8 & - & - \\
2011 & 82 & 11,7 & - & - \\
2012 & 141 & 15,7 & - & - \\
2013 & 99 & 8,3 & - & 8,33 \\
2014 & 115 & 9,6 & 1 & - \\
2015 & 100 & 14,3 & - & - \\
2016 & 100 & 14,3 & - & - \\
2017 & 5 & 1,7 & - & - \\
2018 & 4 & 2 & - & 12,5 \\
2019 & 61 & 7,6 & - & - \\
2020 & 34 & 5,7 & 2,11 \\
\hline Total & 956 & 10,1 & - \\
\hline
\end{tabular}

Fonte: Ministério da Saúde - Sistema de Informações Hospitalares do SUS (SIH/SUS) (2021).

Quando analisado o caráter de atendimento, todas as internações foram configuradas como caráter de urgência. O único estabelecimento onde ocorrera as internações citado fora o Hospital São Lucas.

Apesar da relevância dos estudos relativos a transtornos mentais e comportamentais devido ao uso de álcool no município de Patos de Minas, ainda existem poucos estudos sobre o perfil de internações, dificultando um maior aprofundamento no tema.

Embora esses dados sejam de fundamental importância, ressalta-se que os estudos que utilizam dados secundários possuem algumas limitações, devendo-se ter cautela na interpretação dos resultados, visto que podem ocorrer falhas na codificação das internações, preenchimento inadequado das autorizações, além de múltiplas internações por um mesmo indivíduo que podem superestimar os dados. 


\section{Discussão}

O álcool é uma substancia com propriedades psicoativa capazes de causa dependência. Apesar de ser licito e seu consumo ser difundido em diversas culturas, ele tem um caráter nocivo capaz de causar inúmeras doenças, que além de serem prejudiciais ao indivíduo, tem um grande peso social e é oneroso para o estado (Organização Pan-Americana de Saúde [OPAS], 2020).

Dessa forma, de acordo com a OPAS, o consumo de álcool é associado a mais de 200 doenças, podendo ser relacionado à cirrose hepática, alguns tipos de canceres, doenças cardiovasculares, acidentes de trânsito e, por fim, distúrbios mentais e comportamentais. Sob essa perspectiva, o presente estudo pretende focalizar nas internações decorrentes desses distúrbios psicoativos (OPAS, 2021).

Nesse interim, sabe-se que diversos fatores, seja em nível individuo ou coletivo, alteram os padrões de consumo do álcool, e tal modo, e suas consequências. Podendo estar relacionado a elementos como a disponibilidade, as políticas públicas existentes e ao nível social, sendo o mais relevante a característica de quanto maior o grau de vulnerabilidade, maior a probabilidade do desenvolvimento de problemas decorrentes do álcool. Em virtude disso, houve uma análise do perfil demográfico mais prevalente que se enquadra nos dados obtidos nesse estudo. Sendo possível observar a prevalência do maior número de internações na faixa etária de 40 a 49 anos, seguida pela de 30 a 39 anos. Além disso, outro fator associado é quanto ao sexo de maior incidência, sendo ele o masculino (Garcia \& Sanchez, 2020).

Ademais, procurou-se entender a visão cultural do seu consumo na sociedade hodierna que tem como consequência os dados anteriormente mencionados. O álcool é encontrado em todo decorrer da história, mas seu uso alterou-se de contexto ao longo do tempo. Sob essa ótica, no passado ele era associado ao conceito ritualístico, sendo transformado ao decorrer do tempo e assumindo o contexto atual de diversão, busca pelo prazer e fuga da realidade. Concomitante a isso, alterou-se também o padrão da quantidade e frequência a qual o álcool é consumido, resultando em um consumo de maior periodicidade e dosagem. (Ronzani \& Furtado, 2010)

Diante da perspectiva contemporânea do consumo de álcool, pode-se analisar a quantidade de internações obtidas nesse estudo, que durante o período analisado, ocorreu um total de 95 internações, tendo como pico os anos de 2010, 2013 e 2014. E apesar dos números relativamente baixos nos últimos anos, o presente momento vivido durante a pandemia do COVID-19 alavanca o risco de aumento dos números, uma vez que pesquisas realizadas mostraram que houve um aumento de consumo de álcool em 18\% da população e que $42 \%$ dos entrevistados relataram alto consumo de álcool. (Queiroga et al., 2021)

As internações por transtornos mentais e comportamentais além dos relacionados ao uso de álcool, abordado no presente estudo, inclui também as demências, os transtornos devido ao uso de outras substâncias psicoativas, esquizofrenia, os transtornos de humor, transtornos neuróticos, relacionados com estresse e somatoformes, retardo mental, entre outras causas. Nesse sentido, um estudo realizado em Pernambuco aponta que os transtornos mentais e comportamentais devidos ao uso do álcool encontra-se em segundo lugar em relação ao número de internações e sexto lugar em relação à média de permanência . No entanto, na área de abrangência da $4^{\mathrm{a}}$ Coordenadoria Regional de Saúde ( $\left.4^{\mathrm{a}} \mathrm{CRS}\right)$ a maior demanda de internações foi de transtornos mentais ou comportamentais derivados do uso de álcool e outras drogas, sendo a esquizofrenia o terceiro com maior prevalência (Carvalho et al., 2019).

Ademais, a Lei $\mathrm{n}^{\circ} 148$, de 12 de janeiro de 2012, em seu artigo $3^{\circ}$ indica que o serviço hospitalar de referência para atenção a pessoas com sofrimento ou transtorno mental e com necessidades de saúde decorrentes do uso de álcool, crack e outras drogas construirá seu projeto técnico considerando as internações de curta duração, até a estabilidade clínica do usuário, 
Research, Society and Development, v. 11, n. 1, e19311124640, 2022

(CC BY 4.0) | ISSN 2525-3409 | DOI: http://dx.doi.org/10.33448/rsd-v11i1.24640

respeitando as especificidades de cada caso (Ministério da Saúde [MS], 2012). Nesse sentido, justifica-se o atendimento de urgência de todos os casos de internação encontrados no presente estudo e a liberação após o devido tratamento.

Outrossim, um dos desafios encontrados no cuidado psicossocial é a implementação dos serviços de base comunitária, que atendem também os usuários de álcool (Assis et al., 2014). Nesse sentido, como forma de ampliar esses serviços em 2011 o Ministério da Saúde instituiu a REDE de Atenção Psicossocial (RAPS) que é composta por atenção primária, atenção psicossocial especializada, atenção de urgência e emergência, atenção residencial de caráter transitório, atenção hospitalar e estratégias de desinstitucionalização e reabilitação psicossocial (Ministério da Saúde [MS], 2011). Logo, reduzindo a permanência média hospitalar nos transtornos decorrentes do uso de álcool e garantindo uma atenção continuada ao paciente. Nesse ínterim, observa-se que o tempo de permanência variou entre 215 e 4 dias o que dificulta determinar o perfil das internações na cidade de Patos de Minas, interferindo na implementação de políticas públicas efetivas para a população. No entanto, se tratando de dias de permanência hospitalar, apesar de haver variação, a proporção geral apresenta tendência de queda em relação ao primeiro ano analisado no estudo. Logo, é possível relacionar essa queda a um atendimento mais efetivo ou até mesmo a casos de transtornos mentais e comportamentais considerados mais leves.

Concomitante aos dias de permanência, é relevante abordar os casos de óbitos ocorridos relacionados as internações estudadas. Dessa forma, é possível observar um baixo índice de mortalidade relatado na base de dados fornecida pelo SUS. Vale ressaltar que foram notificados dois casos de óbitos, sendo eles em 2014 e 2019, com taxa de mortalidade relativa a esses anos de 8,33 e 12,5. Todavia, apesar do baixo número de óbitos coletados nesse estudo, vale ressaltar que segundo Garcia, Freitas, Gawryszewski e Duarte (2015) considerar apenas esse número significaria ignorar que a suspensão do consumo de álcool implicaria na completa eliminação de mais de 18 mil mortes por ano, aproximadamente 16 mil de homens e 2 mil de mulheres.

Ademais, os valores totais gastos com serviços hospitalares no período de 2010 a 2020 apresentou grande variação, o que dificulta justificar o perfil de gastos com transtornos mentais e comportamentais causados pelo álcool. No entanto, informações, do presente estudo, sobre os anos 2010 e 2011, demonstraram uma redução significativa de R\$6195,13, assim como o estudo realizado por ), que também apresentou diminuição dos gastos nesses anos. Nesse sentido, o menor valor justifica-se no contexto da Medida Provisória n ${ }^{\circ}$ 498, de 28 de julho de 2010, na qual recursos de 90 milhões de reais foram destinados ao Ministério da Saúde e 35 milhões de reais para a Secretaria Nacional sobre Drogas, para ações em saúde relacionadas ao crack, álcool e outras drogas.

Um estudo transversal encontrou aumento no consumo de bebidas alcoólicas no período de 24 de abril a 24 de maio de 2020, em consequência do distanciamento social durante a pandemia de Covid-19. Nesse sentido, o maior consumo de álcool justificaria o acréscimo no valor gasto nos serviços hospitalares no ano de 2020 em relação ao ano de 2019, no entanto, a média de dias de permanência no ano de 2020 é menor que média de permanência no ano de 2019, o que dificulta justificar o maior gasto em 2020.

Assim como encontrado por ) e Cirilo et al. (2020), o presente estudo possuiu limitações devido as reinternações e transferências do mesmo paciente não serem identificadas no DATASUS, que resulta em contagens cumulativas e supervalorização dos gastos. Ademais, não foi realizado o cálculo para ajustes inflacionários dos gastos nos anos estudados, o que pode interferir no aumento ou diminuição dos valores gastos, contribuindo com a grande variação dos valores encontrados. No entanto, os resultados encontrados permitem visualizar o perfil da morbidade da população em relação aos transtornos mentais e comportamentais devido ao uso de álcool, avaliando o impacto para a população e para a saúde pública. Logo, o uso de sistemas de informação facilita a obtenção de dados, sendo essencial para colaborar na compreensão da situação de saúde da população, contribuindo para o estabelecimento de políticas em todos os níveis de atenção, através do fornecimento de informações úteis para a gestão e o planejamento no SUS (Miranda et al., 2016). 


\section{Conclusão}

É indiscutível que o álcool é um problema de saúde pública no município de Patos de Minas. Realizar esse estudo epidemiológico e caracterizar a parcela populacional que representa o número mais expressivo de internações por transtornos mentais e comportamentais, em razão de seu uso, é um passo crucial para a criação e determinação de estratégias e políticas públicas. Assim, será possível desacelerar esses números que estão em ascensão.

Conforme fora demonstrado, a população que mais foi internada foram os homens, em idade economicamente ativa. Dessa forma, as políticas públicas municipais devem ter um enfoque nesse público, através de campanhas de conscientização, de identificação e intervenção precoce relacionadas ao uso de álcool.

Um outro ponto bastante necessário é a maior adequação do tratamento disponibilizado. No estudo foi possível identificar várias falhas na gestão de dados dessas internações. Os dados devem ser colhidos de formas mais especificada; os dias internados pelo paciente devem ser melhor detalhados e justificados para que se possa identificar um parâmetro e com isso tentar minimizar a reincidência.

Sobreleva mencionar que, quando o Município atinge mais ativamente a população alvo e diminui o uso do álcool, outras esferas socias, além da saúde, melhoram concomitantemente. Um exemplo é a social, que deixará de pagar impostos que serão direcionados para internação e terá seus trabalhadores produzindo e não abandonando seus trabalhos.

Feitas essas considerações, é importante dar ênfase aos estudos epidemiológicos no município de Patos de Minas/MG. Isso possibilita sistematizar e gerir melhor as internações por transtornos mentais e comportamentais proveniente do álcool, além de direcionar o Poder Público e os órgãos de Saúde sobre qual é a parcela da população que será mais direcionada às campanhas, investimento e políticas públicas. Dessa forma, torna-se relevante que possíveis trabalhos futuros realizem uma pesquisa referente aos tratamentos e abordagem destinadas aos pacientes internados por transtornos mentais e comportamentais devido ao uso de álcool, bem como a eficiência de políticas públicas sobre o público alvo.

\section{Referências}

American Psychiatrist Association. (2014). Manual de Diagnóstico e Estatístico de Transtornos Mentais (5 ${ }^{a}$ ed). Porto Alegre: Artmed. http://www.niip.com.br/wp-content/uploads/2018/06/Manual-Diagnosico-e-Estatistico-de-Transtornos-Mentais-DSM-5-1-pdf.pdf.

Assis, J. T., Barreiros, C. A, Jacinto, A. B. M., Kinoshita, R.T., Macdowell, P. L., Mota, T. D., Nicácio, F., Schorn, M. C., Souza, I. S. \&Trino, A. T. (2014). Política de saúde mental no novo contexto do Sistema Único de Saúde: regiões e redes. Saúde em Debate, 52(ISSN0103-4383), 88-113. http://cebes.org.br/site/wp-content/uploads/2014/12/Divulgacao-52.pdf.

Carvalho, K. L., Terra, M. G., Moreschi, C., Siqueira, D. F., Mello, A. L. \& Alessandra Gamermann, A. (2019).

Características de internações em saúde mental de hospitais gerais do Rio Grande do Sul. Revista mineira de enfermagem, 23(e-1203), 1-8. https://cdn.publisher.gn1.link/reme.org.br/pdf/e1203.pdf. Doi: 10.5935/1415-2762.20190051.

Castro, D. S. (2014). Consumo de álcool no padrão “beber pesado episódico”: um levantamento nacional. Tese de Doutorado, Universidade Federal de São Paulo, São Paulo, SP, Srasil. https://repositorio.unifesp.br/bitstream/handle/11600/46198/Daniel\%20S\%c3\%b3crates\%20de\%20Castro.pdf?sequence=1\&isAllowed=y.

Cirilo, M. V. S., Dantas, G. B., Freitas, J. B. S, Arantes, B. O., Vieira, G. H, Paniago, G. H., Silva, K. L. F, Laureano, K. G., Herbas, M. A. M., Carvalho, A. A, Cardoso, K. S. \& Abdala, C. C. (2020). Gastos com serviços hospitalares relacionados aos transtornos mentais e comportamentais devido ao uso de substâncias psicoativas no Brasil: uma comparação entre os anos de 2009 a 2019. Condições teórico-práticas das ciências da saúde no Brasil, 3 , 147-157. https://sistema.atenaeditora.com.br/index.php/admin/api/artigoPDF/43183. doi: 10.22533/at.ed.39320031215.

Coelho, R. C. B. \& Parente, A. S. (2019). Perfil de internações por transtornos mentais e comportamentais no Estado de Pernambuco. Revista de psicologia, 13(46), 24-32. https://idonline.emnuvens.com.br/id/article/view/1803. Doi: https://doi.org/10.14295/idonline.v13i46.1803.

Garcia L. P. \& Sanchez, Z. M. (2020). Consumo de álcool durante a pandemia da COVID-19: uma reflexão necessária para o enfrentamento da situação. Cadernos de Saúde Pública, 36(10), e00124520. https://www.scielo.br/j/csp/a/FbtYqzqTP35S8qhYxqhhrVc/?format=pdf\&lang=pt. Doi: 10.1590/0102$311 \mathrm{X} 00124520$

Garcia L. P., Freitas L. R. S., Gawryszewski V. P. \& Duarte E. C. (2015) Uso de álcool como causa necessária de morte no Brasil, 2010 a 2012. Revista Panamericana de Salud Pública, 38(4), 418-424. https://www.scielosp.org/pdf/rpsp/2015.v38n5/418-424/pt.

Malta D. C., Gomes C. S., Szwarcwald C. L., Barros M. B. A., Silva A. G., Prates E. J. S., Machado I. E., Souza Júnior P. R. B., Romero D. E., Lima M. G., 
Research, Society and Development, v. 11, n. 1, e19311124640, 2022

(CC BY 4.0) | ISSN 2525-3409 | DOI: http://dx.doi.org/10.33448/rsd-v11i1.24640

Damacena G.N., Azevedo L.O., Pina M.F., Werneck A.O. \& Silva D.R.P. (2021). Distanciamento social, sentimento de tristeza e estilos de vida da população brasileira durante a pandemia de Covid-19. Saúde em http://www.scielo.br/j/sdeb/a/8YsdKcVzwf3yYVZqWMnbnXs/abstract/?lang=pt. Doi:10.1590/0103-11042020E411.

Ministério da Saúde. (2012). Gabinete do Ministro. Portaria $N^{o} \quad 148$, de $31 \quad$ de Janeiro de 2012. https://bvsms.saude.gov.br/bvs/saudelegis/gm/2012/prt0148_31_01_2012.html.

Ministério da Saúde. (2011). Gabinete do Ministro. Portaria $n^{o} \quad 3.088$, de 23 de dezembro de 2011. https://bvsms.saude.gov.br/bvs/saudelegis/gm/2011/prt3088_23_12_2011_rep.html.

Miranda, S. S., Martins, E. M., Queiroz, L. A., Andrade, A. P. E. do N., Santos, L. P. S., Sodré, T. M., \& Oliveira, L. B. de. (2017). Os sistemas de informação em saúde e seu apoio à gestão e ao planejamento do Sistema Único de Saúde: análise de um município de médio porte da região Nordeste. Revista Brasileira De Pesquisa Em Saúde, 18(4), 14-21. https://periodicos.ufes.br/rbps/article/view/16725.

Organização Pan-Americana de Saúde. (2020). Álcool. https://www.paho.org/pt/topicos/alcool.

Organização Pan-Americana de Saúde. (2021). Cerca de 85 mil mortes a cada ano são $100 \%$ atribuídas ao consumo de álcool nas Américas, constata estudo da OPAS/OMS. https://www.paho.org/pt/noticias/12-4-2021-cerca-85-mil-mortes-cada-ano-sao-100-atribuidas-ao-consumo-alcool-nas-americas.

Pesquisa nacional de saúde. (2019). Percepção do estado de saúde, estilos de vida, doenças crônicas e saúde bucal: Brasil e grandes regiões. Coordenação de Trabalho e Rendimento. Rio de Janeiro: IBGE. https://www.pns.icict.fiocruz.br/wp-content/uploads/2021/02/liv101764.pdf.

Queiroga, V. V., Filgueira, E. G. K., Vasconcelos, A. M. de A., Procópio, J. V. V., Gomes, F. W. C., Gomes, C. H. F. de M., Gomes Filho, C. A. M., Jacó, A.P., Araujo, J. M. B. G., Nóbrega, J. C. da S., \& Nóbrega Filho, M. M. de S. (2021). A pandemia da Covid-19 e o aumento do consumo de álcool no Brasil. Research, Society and Development, 10(11), e568101118580. https://doi.org/10.33448/rsd-v10i11.18580.

Rocha, F. V., Oliveira, R. L., Brum, D. A. S., Cavalcante, R. B. \& Machado, R. M. (2015). Epidemiologia dos transtornos do desenvolvimento psicológico em adolescentes: uso de álcool e outras drogas. Revista Rene, 16(1), 54-63. http://www.periodicos.ufc.br/rene/article/view/2663. Doi: 10.15253/21756783.2015000100008

Ronzani, T. M. \& Furtado, E. F. (2010). Estigma social sobre o uso de álcool. Jornal brasileiro de psiquiatria, 59(4), 326-332. https://www.scielo.br/j/jbpsiq/a/G4C8v9mqySmQRgNdy8QZbjf/?lang=pt. Doi: https://doi.org/10.1590/S0047-20852010000400010.

World Health Organization (WHO). (2018). Global Status Report on Alcohol and Health. https://www.who.int/publications/i/item/9789241565639.

Zurita, R. C. M., Melo, E. C., Oliveira, R. R., Latorre, M. R. D. O. \& Mathias, T. A. F. (2016). Evolução dos gastos hospitalares com internações psiquiátricas por drogas. Revista Gaúcha de Enfermagem, 37(3), e53289. https://www.scielo.br/j/rgenf/a/bWf3XyqjQpfgXB4TFC9sVyL/?format=pdf\&lang=pt. Doi: $10.1590 / 1983-1447.2016 .03 .53289$ 